\title{
Discrete set-valued continuity and interpolation
}

\author{
Laurent Najman ${ }^{1}$ and Thierry Géraud ${ }^{2}$ \\ 1 Université Paris-Est, LIGM, Équipe A3SI, ESIEE \\ 2 EPITA Research and Development Laboratory (LRDE) \\ 1.najman@esiee.fr, thierry.geraud@lrde.epita.fr
}

\begin{abstract}
The main question of this paper is to retrieve some continuity properties on (discrete) T0-Alexandroff spaces. One possible application, which will guide us, is the construction of the so-called "tree of shapes" (intuitively, the tree of level lines). This tree, which should allow to process maxima and minima in the same way, faces quite a number of theoretical difficulties that we propose to solve using set-valued analysis in a purely discrete setting. We also propose a way to interpret any function defined on a grid as a "continuous" function thanks to an interpolation scheme. The continuity properties are essential to obtain a quasi-linear algorithm for computing the tree of shapes in any dimension, which is exposed in a companion paper [10].
\end{abstract}

\section{Introduction}

This paper is the first in a series dedicated to the notion of the tree of shapes, which has been introduced $[16,7]$ as a way to filter an image $u: X \subset \mathbb{R}^{n} \rightarrow \mathbb{R}$ $(n \geq 2)$ in a self-dual way, meaning intuitively that processing either $u$ or $-u$ would give the same result. In its continuous definition, this tree is made by the connected components of upper level sets $\{x ; u(x) \geq \lambda\}_{\lambda \in \mathbb{R}}$ and lower level sets $\{x ; u(x)<\lambda\}_{\lambda \in \mathbb{R}}$. This definition, by itself, is not really self-dual, as the tree of shapes of $u$ is not the same as the tree of shapes of $-u$.

The goal of this paper is to propose a purely discrete framework in which a true self-dual definition of the tree of shapes can be given, together with a proof of the existence and uniqueness of such a tree for a given class of functions. We then provide several ways to interpret any image as belonging to this class of functions, one of them being self-dual. In the next paper of this series [10], we provide a quasi-linear algorithm relying on this theoretical framework, that allows the computation of the tree of shapes of an image, whatever its dimension.

In order to achieve our goal, we extend the notion of set-valued uppersemicontinuity [4] to the discrete case (T0-Alexandroff topology), and from which we build the notions of simple map and plain map that share the main properties of a classical continuous function. To obtain the tree of shapes, we also need to adapt the notion of well-composed map to our framework.

First approaches to discrete continuity date back to Rosenfeld [19], followed by Boxer [6]. Many authors have recognized that the set-valued setting is important to the discrete case $[12,22,8]$. None has proposed what we develop in this paper, although it may appear very natural for a researcher familiar with set-valued analysis. 


\section{Set-valued continuity on discrete spaces}

\subsection{Topology reminder}

A topological space is a set $X$ composed of elements, or points, of arbitrary nature in which certain subsets $A \subseteq X$, called closed sets of the topological space $X$, have been defined so as to satisfy the following conditions, called the axioms of a topological space:

1. The intersection of any number and the union of any finite number of closed sets is a closed set.

2. The whole set $X$ and the empty set $\emptyset$ are closed.

The sets complementary to the closed sets of $X$ are called the open sets of the topological space $X$.

The intersection of all closed sets containing a set $M \subset X$ is called the closure of $M$ in the topological space $X$ and is denoted by $c l_{X}(M)$. Every open set containing a set $M$ is called a neighborhood of the set $M$. A set $M \subset X$ is said to be degenerate if it contains just one point.

Definition 1 A topological space $X$ is said to be a $T_{0}$-space if every two distinct degenerate subsets of $X$ have distinct closures in $X . A T_{0}$-space is called a discrete space if the union of an arbitrary number of closed sets of the space is closed.

Finite $T_{0}$-spaces are the most important cases of the so called discrete spaces $[1-$ 3]. Another important example is the Khalimsky grid of dimension $n$, which is define as follows:

$$
\begin{aligned}
& H_{0}^{1}=\{\{x\} ; x \in \mathbb{Z}\} \\
& H_{1}^{1}=\{\{x, x+1\} ; x \in \mathbb{Z}\} \\
& H^{1}=H_{0}^{1} \cup H_{1}^{1} \\
& H^{n}=\left\{h_{1} \times \ldots \times h_{n}, \forall i \in[1, n], h_{i} \in H^{1}\right\}
\end{aligned}
$$

In the Khalimsky grid, the elements of $H_{0}^{1}$ are closed sets, and the elements of $H_{1}^{1}$ are open sets. Although our definitions and results are valid in any discrete space, we will illustrate them on (a subset of) the Khalimsky grid.

If $X$ is a discrete space, the intersection of all open sets containing a set $M \subset X$ is an open set, called the star of $M$ in the topological space $X$. The star of $M$ in $X$ is denoted by $s t_{X}(M)$. If $M=\{x\}$ is degenerate, by abuse of notation, we write $s t_{X}(x)=s t_{X}(\{x\})$.

Definition $2 A$ set is said to be connected if it is not the union of two disjoint nonempty closed sets.

For discrete spaces, this definition is equivalent to the one provided by the connectivity by paths. Two points $x$ and $y$ of $X$ of a discrete space $X$ are neighbors if either $y \in s t_{X}(x)$ or $x \in s t_{X}(y)$. A path in $X$ from $x$ to $y$ is a sequence 
$x_{0}=x, x_{1}, \ldots, x_{n}=y$ such that $x_{i+1}$ is a neighbor of $x_{i}$. One can prove that a set is connected if for any two points of this set, there exists a path between them. A connected component of $X$ is a connected subset of $X$ that is maximal for the connectivy property. We can also mention that any discrete space is locally connected, in the sense that any point $x \in X$ has a smallest open connected neighborhood in $X$, namely $s t_{X}(x)$. The following lemma is useful in the sequel.

Definition 3 Let $M$ and $N$ be two subspaces of $X . M$ and $N$ are separated if each is disjoint from the closure of the other, that is, if $\left(M \cap c l_{X}(N)\right) \cup(N \cap$ $\left.c l_{X}(M)\right)=\emptyset$.

Lemma 4 If two open sets of a discrete space are disjoint, then they are separated.

\subsection{Set-valued maps}

In the sequel, $X$ and $Y$ denotes two discrete spaces. The main references for set valued analysis is [4]. An application $F$ is called a set-valued map from $X$ to $Y$ if for any $x \in X, F$ associates to $x$ the set $F(x) \subset Y$, called the image of $F$ at $x$. In this case, the domain of $F$ is the set $\operatorname{Dom}(F)=\{x: F(x) \neq \emptyset\}$.

A set-valued map $F: X \rightsquigarrow Y$ is called upper semicontinuous at $x \in \operatorname{Dom}(F)$ (USC at $x$ ) if and only if for all $y \in s t_{X}(x), F(y) \subseteq s t_{Y}(F(x)) . F$ is said to be upper semicontinuous if and only if it is upper semicontinuous at every point $x \in \operatorname{Dom}(F)$.

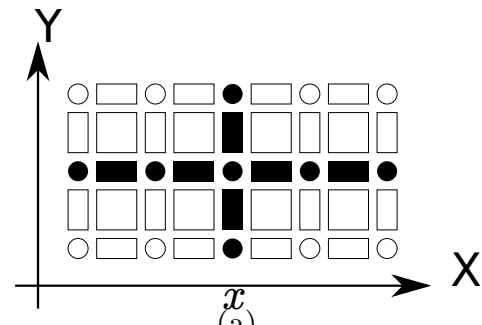

(a)

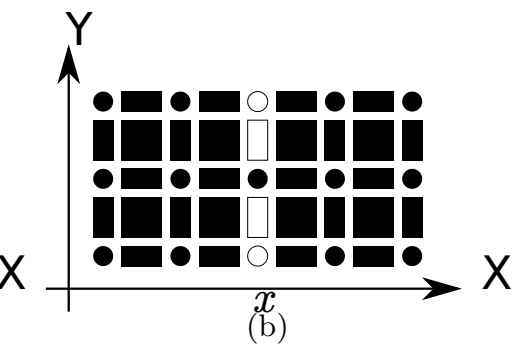

(b)

Fig. 1. (a) A discrete set-valued map $F$ that is USC at $x$, drawn on a subset of the Khalimsky grid $H_{2}$ Note that $F$ is also an interval-valued map, and $x$ is both a minimum and a maximum of $F$. (b) A discrete set-valued map that is LSC at $x$.

One can remark that upper semicontinuity is the natural adaptation to setvalued map of the definition of a continuous function. An example of a USC set-value map is given in Fig. 1(a).

A useful property of a continuous function is that the inverse image of a closed (open) set is a closed (open) set. To adapt this property to set valued maps, we need to define the inverse. Let $M$ be a subset of $Y, F: X \rightsquigarrow Y$ 
be a set-valued map. We denote $F^{\oplus}(M)=\{x \in X ; F(x) \cap M \neq \emptyset\}$, and $F^{\ominus}(M)=\{x \in X ; F(x) \subseteq M\}$. The subset $F^{\oplus}(M)$ is called the inverse image of $M$ by $F$ and $F^{\ominus}(M)$ is called the core of $M$ by $F$.

Proposition 5 A set-valued map $F: X \rightsquigarrow Y$ is USC if and only if the core of any open set is open: $F$ is USC if and only if $F^{\ominus} s t_{Y}=s t_{X} F^{\ominus}$

If furthermore $F$ is with nonempty values (or if $\operatorname{Dom}(F)$ is closed) $F$ is USC if and only if the inverse image of any closed subset is closed: $F$ is USC if and only if $F^{\oplus} c l_{Y}=c l_{X} F^{\oplus}$.

Intuitively, a function is continuous if, roughly speaking, it can be drawn as a single unbroken curve with no "holes" nor "jumps". In other words, a continuous function transforms a connected set into a connected set. For a set $M \subseteq X$, we write $F(M)=\cup_{x \in M} F(x)$.

Proposition 6 Let $F$ be a USC set-valued map such that, for all $x \in X, F(x)$ is a closed (resp. open) connected set. Then, for any connected set $M, F(M)$ is a closed (resp. open) connected set.

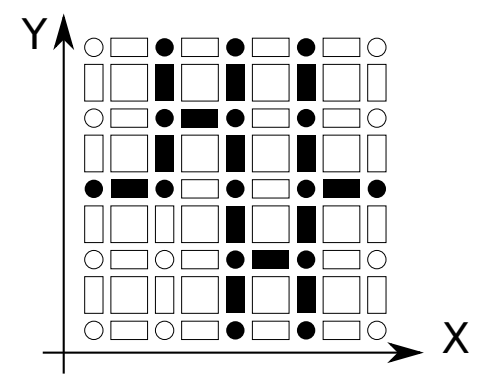

Fig. 2. A quasi-simple map.

We say that a USC set-valued map $F$ is a closed (resp. open) quasi-simple map if for all $x \in X, F(x)$ is a closed (resp. open) connected set, and if furthermore, for any $\{x\}=s t_{X}(x) \in X, F(x)$ is degenerate. A quasi-simple map $F$ is simple if it is the smallest of all quasi-simple maps with the same data on open points, i.e, a quasi-simple map $F_{1}$ is simple if for any quasi-simple map $F_{2}$ such that for any $\{x\}=s_{X}(x) \in X, F_{1}(x)=F_{2}(x)$, then, for all $x \in X, F_{1}(x) \subseteq F_{2}(x)$. Fig. 2 is an example of a quasi-simple map, while Fig. 3 is a simple map. The class of simple maps is interesting because it corresponds to our intuition (i.e. a single ubroken curve with no "holes" nor "jump") of continuous functions (at least when a simple map is interval-valued, see section 3 ).

In particular if $f$ is a function defined for all $\{x\}=s t_{X}(x) \in X$ with $f(x) \in Y$ and $f(x)$ closed (resp. open), there exists a (unique) closed (resp. open) simple map such that for all $\{x\}=s t_{X}(x) \in X, F(x)=\{f(x)\}$. 
Remark 1. V. Kovalevsky [12] defines the "continuous" functions as the ones that are called lower semicontuinous in our framework. More precisely, V. Kovalevsky uses prop. 8 below as the definition of a continuous function.

Definition 7 A set-valued map $F: X \rightsquigarrow Y$ is called lower semicontinuous (LSC) at $x \in \operatorname{Dom}(F)$ if for any open subset $M \subset Y$ such that $M \cap F(x) \neq \emptyset$, then for all $y \in \operatorname{st}_{X}(x), F(y) \cap M \neq \emptyset$.

Fig. 1(b) gives an example of an LSC set-valued map. This definition is the adaptation to the (discrete) set-valued setting of the celebrated characterization of continuous function (a function $f$ is continuous at $x$ if and only if it maps any sequence converging to $x$ to sequences converging to $f(x)$.) However, as no convergence can be defined in a discrete space, we have to use a topological definition. We have the following characterization of LSC maps.

Proposition 8 A set-valued map $F: X \rightsquigarrow Y$ is LSC if and only if the inverse image of any open set is open: $F$ is LSC if and only if $F^{\oplus} s t_{Y}=s t_{X} F^{\oplus}$

If furthermore $F$ is with nonempty values (or if $\operatorname{Dom}(F)$ is closed) $F$ is LSC if and only if the core of any closed subset is closed: $F$ is LSC if and only if $F^{\ominus} c l_{Y}=c l_{X} F^{\ominus}$.

However, it is not possible to obtain simple LSC set-valued maps with interesting properties for our goal, and V. Kovalevsky then studies connectivity-preserving set-valued maps, that would be called Darboux maps in a more classical setting. In our framework (as in the classical one), some connectivity-preserving maps exist that are not USC, although prop. 6 shows that there exists USC maps preserving connectivity.

\section{Interval-valued maps}

Recall that a set $X$ is said to be unicoherent if $X$ is connected and for any two closed connected sets $M$ and $N$ of $X$ such that $X=M \cup N$, then $M \cap N$ is connected. In the sequel, $X_{0}$ denotes a finite discrete topological space, and $X \subset X_{0}$ denotes an unicoherent subset of $X_{0}$.

An interval is a connected subset of $H^{1}$. Remark that $H^{1}$ is a totally ordered set, in the sense where $H^{1}$ is in bijection with $\frac{1}{2} \mathbb{Z}$ : we identify the open sets of $H^{1}$ with the elements of $\frac{1}{2} \mathbb{Z} \backslash \mathbb{Z}$ and the closed sets of $H^{1}$ with the elements of $\mathbb{Z}$. We thus have $\ldots<\{0\}<\{0,1\}<\{1\}<\ldots$, and operations such as $\lambda+\frac{1}{2}$ (where $\lambda \in H^{1}$ ), max or min on any subset of $H^{1}$ are well-defined. We say that a set-valued map $F$ is an interval-valued map if the images of $F$ are intervals. We say that an interval-valued map is bounded if there exists $\lambda$ and $\mu$ in $H^{1}$ such that $\lambda \leq \min \{\nu \in F(X)\}$ and $\mu \geq \max \{\nu \in F(X)\}$. It is easy to check that interval-valued maps verify important classical theorems, such as for example, the intermediate value theorem (which is a simple consequence of Pr. 6 and of the connectedness of an interval).

As illustrated on Fig. 3, a simple closed-valued interval-valued map from $H^{1}$ to $H^{1}$ is what one would intuitively draw when dealing with a classical continuous function. Thus we will use the following definition. 
Definition 9 A closed-valued, interval-valued simple map $F$ from $X$ to $H^{1}$, with $\operatorname{dom}(F)=X$ is called a plain map on $X$.

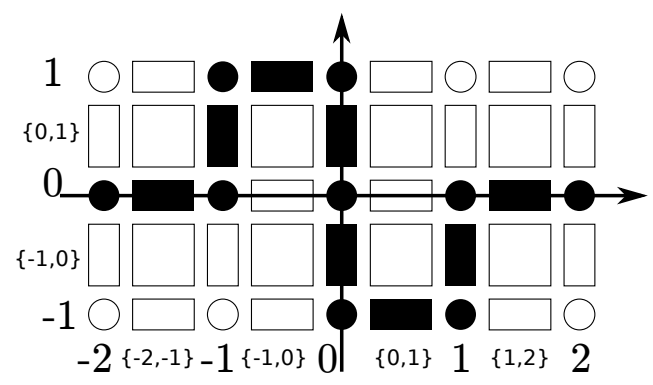

Fig. 3. A plain map $F$ from $H^{1}$ to $H^{1}$.

Remark that, as $X$ is finite, a plain map is bounded. We have the following property.

Proposition 10 If $F$ is a plain map on $X$, then $F^{\ominus}$ is an open-valued USC map and $F^{\oplus}$ is a closed-valued USC map.

\subsection{Level sets and extrema}

We need to adapt to the set-valued case the notions of upper and lower level sets. In the sequel, $F$ denotes an interval-valued map from $X$ to $H^{1}$. Let $\lambda \in \mathbb{Z}$, we write $[F \triangleleft \lambda]=F^{\ominus}(]-\infty, \lambda[)=\{x \in X \quad \mid \forall \mu \in F(x), \mu<\lambda\}$. The set $[F \triangleleft \lambda]$ is called the (strict) lower level set (of $F$, at level $\lambda$ ). Similarly, the (strict) upper level set (of $F$, at level $\lambda$ ) is the set $[F \triangleright \lambda]=F^{\ominus}(] \lambda,+\infty[$ ). Thanks to prop. 10, the upper and lower level sets of a plain map are open.

The connected components of a set $M \subseteq X$ will be denoted by $\mathcal{C C}(M)$. If $x \in M$, the connected component of $M$ that contains $x$ will be denoted by $\mathcal{C C}(M, x)$, and by extension, we write $\mathcal{C C}(M, x)=\emptyset$ if $x \notin M$. If $\emptyset \neq C \subseteq M$ and $C$ is connected, the connected component of $M$ containing $C$, denoted by $\mathcal{C C}(M, C)$ is $\mathcal{C C}(M, x)$ with $x \in C$.

Definition 11 (Extrema) A connected component of $[F \triangleleft \lambda]$ is a minimum of $F$ if it does not contain any other connected component of $[F \triangleleft \mu]$ for any $\mu<\lambda$. A connected component of $[F \triangleright \lambda]$ is a maximum of $F$ if it does not contain any other connected component of $[F \triangleright \mu]$ for any $\mu>\lambda$. An extremum of $F$ is either a maximum or a minimum of $F$.

We say that a set $M$ is flat for $F$ if for all $x \in M, F(x)=F(M)$. Remark that $F(M)$ is not always a degenerate set and that an extremum of a set-valued map is not always flat: for example, the point $x$ in Fig. 1(a) is both a minimum 
and a maximum, and although this extremum is flat, it is not degenerate. It is easy to draw an example where a minimum of a set valued map contains several maxima, and hence is not flat.

Lemma 12 The extrema of a plain map $F$ are flat open sets. Furthermore, for any extrema $M$ of $F, F(M)$ is degenerate.

Proposition 13 Let $F$ be a plain map on $X$ that is not constant. Then the extrema of $F$ are separated.

In the same way as the strict level sets, we can define the (large) lower and upper level sets $\left.\left.[F \unrhd \lambda]=X \backslash[F \triangleleft \lambda]=F^{\oplus}(]-\infty, \lambda\right]\right)=\{x \in X \mid \exists \mu \in$ $F(x) ; \mu \leq \lambda\}$ and $[F \unlhd \lambda]=X \backslash[F \triangleright \lambda]$. Thanks to prop. 10, $[F \unrhd \lambda]$ and $[F \unlhd \lambda]$ of a plain map $F$ are closed.

Combining lower and upper level sets relations, we write $[F \equiv \lambda]=[F \unlhd$ $\lambda] \cap[F \unrhd \lambda]=\{x \in X \mid \lambda \in F(x)\}$ and $[F \not \equiv \lambda]=X \backslash[F \equiv \lambda]=\{x \in$ $X \mid \lambda \notin F(x)\}=[F \triangleleft \lambda] \cup[F \triangleright \lambda]$.

The following relations are natural. We indeed have $[F \unlhd \lambda]=[F \triangleleft \lambda] \cup[F \equiv$ $\lambda],[F \unrhd \lambda]=[F \triangleright \lambda] \cup[F \equiv \lambda]$ and $[F \not \equiv \lambda]=[F \triangleleft \lambda] \cup[F \triangleright \lambda]$. Similarly, we have for any $\lambda_{1}$ and $\lambda_{2}$ in $H^{1}, \lambda_{1}<\lambda_{2} \Rightarrow\left[F \unlhd \lambda_{1}\right] \subseteq\left[F \unlhd \lambda_{2}\right]$ and $\lambda_{1}<$ $\lambda_{2} \Rightarrow\left[F \unrhd \lambda_{2}\right] \subseteq\left[F \unrhd \lambda_{1}\right]$. But if we have $\lambda_{1}<\lambda_{2} \Rightarrow\left[F \triangleleft \lambda_{1}\right] \cap\left[F \triangleright \lambda_{2}\right]=\emptyset$, the following relation is uncommon.

$$
\lambda_{1}<\lambda_{2} \nRightarrow\left[F \unlhd \lambda_{1}\right] \cap\left[F \unrhd \lambda_{2}\right]=\emptyset
$$

Indeed, for instance, for $x$ such as $F(x)=[1,2]$, we have both $x \in[F \unlhd 1]$ and $x \in[F \unrhd 2]$. Note that we may also have:

$$
\left[F \triangleleft \lambda+\frac{1}{2}\right] \varsubsetneqq[F \unlhd \lambda]
$$

Indeed, with the same example, if $F(x)=[1,2], x \in[F \unlhd 1]$, but $x \notin\left[F \triangleleft \frac{3}{2}\right]$. But, by the very definition of a plain map, if $F$ is plain, for any $\lambda \in H^{1}$, we always have the following property.

Proposition 14 A set-valued map $F$ is a plain map if and only if, for any $\lambda \in H^{1}$,

$$
[F \unlhd \lambda]=c l_{X}\left(\left[F \triangleleft \lambda+\frac{1}{2}\right]\right) \text { and }[F \unrhd \lambda]=c l_{X}\left(\left[F \triangleright \lambda-\frac{1}{2}\right]\right)
$$

We can reconstruct $F$ from its lower and upper level sets, with the following process: set $f_{\min }(x)=\min \{\lambda \in \mathbb{Z} ; x \in[F \unlhd \lambda]\}$ and $f_{\max }(x)=\max \{\lambda \in \mathbb{Z} ; x \in$ $[F \unrhd \lambda]\}$. Then $F(x)=\left[f_{\min }(x), f_{\max }(x)\right]$. The process is simpler if $F$ is plain: then for any degenerate open set $x \in X$, we have $F(x)=\{\min \{\lambda \in \mathbb{Z} ; x \in$ $[F \triangleleft(\lambda+1)]\}\}=\{\max \{\lambda \in \mathbb{Z} ; x \in[F \triangleright(\lambda-1)]\}\}$. The other images of a simple/plain map $F$ can be deduced from these ones. 
Definition 15 Let $\mathcal{T} \subseteq 2^{X}$. We say that $\mathcal{T}$ is a tree (of subsets of $X$ ) if (i) $X \in \mathcal{T}$, and (ii), if $M, N \in \mathcal{T}$, then either $M \cap N \neq \emptyset, M \subseteq N, N \subseteq M$. In the last two cases, we say that $M$ and $N$ are nested.

For a plain map $F$, let us denote $\mathcal{U}^{\ominus}(F)=\left\{M ; M \in \mathcal{C C}([F \triangleleft \lambda]), \lambda \in H^{1}\right\}$, $\mathcal{U}^{\oplus}(F)=\left\{M ; M \in \mathcal{C C}([F \unlhd \lambda]), \lambda \in H^{1}\right\}, \mathcal{L}^{\ominus}(F)=\{M ; M \in \mathcal{C C}([F \triangleright \lambda]), \lambda \in$ $\left.H^{1}\right\}$ and $\mathcal{L}^{\oplus}(F)=\left\{M ; M \in \mathcal{C C}([F \unrhd \lambda]), \lambda \in H^{1}\right\}$. We remark that any two elements of one of these four sets are either disjoint or nested, hence they are trees. The set $\mathcal{U}^{\ominus}(F)$ (resp. $\left.\mathcal{L}^{\ominus}(F)\right)$ is the tree of open connected components of the lower (resp. upper) level sets of $F$, while $\mathcal{U}^{\oplus}(F)\left(\operatorname{resp} . \mathcal{L}^{\oplus}(F)\right.$ ) is the tree of closed connected components of the lower (resp. upper) level sets of $F$. In the literature, similar trees have been called min- or max-trees, or component trees.

\section{Shapes of Interval-Valued Maps}

Topology is not so important when we consider independently the min or the max tree. It becomes fundamental when considering both trees at the same time, which is at the basis of the so-called tree of shapes.

Definition 16 Let $M \subseteq X$. We call cavities of $M$ in $X$ the components of $X \backslash M$. Let $p_{\infty} \in X$ a reference point. We call saturation of $M$ with respect to $p_{\infty}$ the set $\operatorname{sat}\left(M, p_{\infty}\right)=X \backslash \mathcal{C C}\left(X \backslash M, p_{\infty}\right)$.

For any $\lambda \in H^{1}$, we call quasi-shape of $x$ the set:

$$
S_{\lambda}(F, x)=\operatorname{sat}\left(\mathcal{C C}([F \not \equiv \lambda], x), p_{\infty}\right)
$$

We thus have two different types possible for a given shape:

$$
\begin{aligned}
\text { either } S_{\lambda}(F, x) & =\operatorname{sat}\left(\mathcal{C C}([F \triangleright \lambda], x), p_{\infty}\right) \\
\text { or } S_{\lambda}(F, x) & =\operatorname{sat}\left(\mathcal{C C}([F \triangleleft \lambda], x), p_{\infty}\right) .
\end{aligned}
$$

But all the shapes being open, the type of a shape can not be known from the shape itself (contrary to the case of the continuous framework [7]).

Consider the family of sets:

$$
\mathfrak{S}(F, x)=\left\{S_{\lambda}(F, x)\right\}_{\lambda} \backslash \emptyset .
$$

and denote by $\mathfrak{S}(F)$ the set formed by all the $\mathfrak{S}(F, x)$ for all $x \in X$.

The notion of surface used in this paper is the one of $n$-surface.

Definition 17 (n-surface, $[\mathbf{5}, \mathbf{9}])$ A discrete space $Y$ is a 0 -surface if $Y$ is made of exactly two points $x$ and $y$ such that $x \notin s t_{Y}(y)$ and $y \notin s t_{Y}(x)$. A discrete space $Y$ is a n-surface $(n>0)$ if $Y$ is connected and if, for any $x \in Y, c l_{Y}(x) \cup$ $s_{Y}(x) \backslash\{x\}$ is a $(n-1)$-surface.

The notion of well-composed connected set has been introduced in [13,14]. Several extensions have been proposed in the literature $[15,11,23]$. In this paper, we extend it to maps by considering at the same time upper and lower level sets. 
Definition 18 (Well-composed set and map) A connected set $M$ is wellcomposed if $\partial M=c_{X}(M) \cap c l_{X}(X \backslash M)$ is a n-surface. A set $N$ is wellcomposed if any connected component of $\partial N$ is a n-surface. A plain map $F$ is well-composed if for any $\lambda \in H^{1}$, both $[F \triangleleft \lambda]$ and $[F \triangleright \lambda]$ are well-composed.

Remark 2. Note that a well-composed set is a regular open set [18].

Lemma 19 If a plain map $F$ is well-composed, then for any $\lambda \in H^{1}$, and for any connected component $C \in \mathcal{C C}([F \unlhd \lambda])$ (resp. $C \in \mathcal{C C}([F \unrhd \lambda])$ ), there exists a connected component $C C \in \mathcal{C C}\left(\left[F \triangleleft \lambda+\frac{1}{2}\right]\right)$ (resp. $\left.C C \in \mathcal{C C}\left(\left[F \triangleright \lambda-\frac{1}{2}\right]\right)\right)$ such that $C=\operatorname{cl}_{X}(C C)$.

Lemma 20 If a plain map $F$ is well-composed, then for any $\lambda \in H^{1}$, and for any connected component $M \in \mathcal{C C}([F \unlhd \lambda])$ (resp. $M \in \mathcal{C C}([F \unrhd \lambda])$ ), we have $\operatorname{sat}\left(\operatorname{cl}_{X}(M)\right)=\operatorname{cl}_{X}(\operatorname{sat}(M))$.

Theorem 21 If $F$ is a well-composed plain map, then any two quasi-shapes are either disjoint or nested. Hence $\mathfrak{S}(F)$ is a tree.

The proof of Th. 21 is essentially the same as the one of Th. 2.6 in [7], adapted for the set-valued case of a plain map, and with a different ending that uses Lemma 20 and Lemma. 19. Due to space constraint, this proof will be provided in an extended version of this paper.

Definition 22 We call shape of $x$ the smallest non-empty quasi-shape of $x$ defined by:

$$
S(F, x)=\bigcap_{S \in \mathfrak{S}(F, x)} S
$$

We denote by $S(F)$ the set formed by all the shapes of $x$ for all $x \in X$

The intersection in (12) is open as an intersection of open sets in a discrete space; moreover it is non-empty, as $S(F, x)$ is one of the quasi-shapes of $x$ by proposition 21 .

Corollary 23 The set $S(F)$ is a tree (called the tree of shapes).

\section{Interpolation}

In order to apply the framework, we need a way to interpret any function defined on a grid as a well-composed plain map. Unfortunately, directly interpreting a set of data as a plain map (as in Fig. 4.a) does not lead to a well-composed plain map. We do not want to modify the original data, as it is done in [20]. Closer to what we propose in this paper are the magnification process of [17], and even closer is [21], where new points are introduced thanks to subdivision of the space, the value on the points being derived from a majority rule. However, none of the previous approaches is equivalent to the simple interpolation we propose 


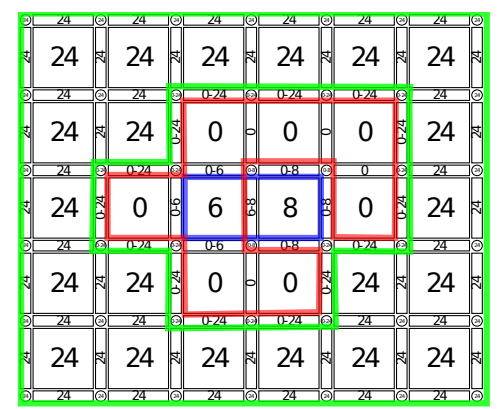

(a) Original data seen as a plain map.

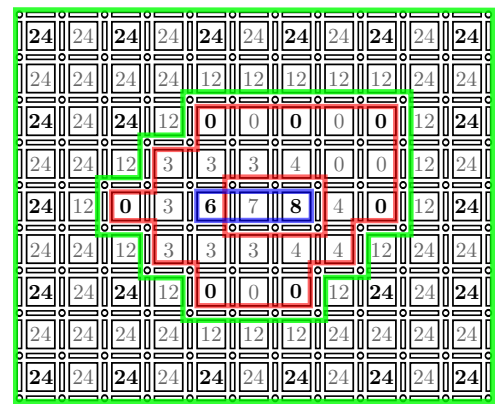

(c) Median interpolation.

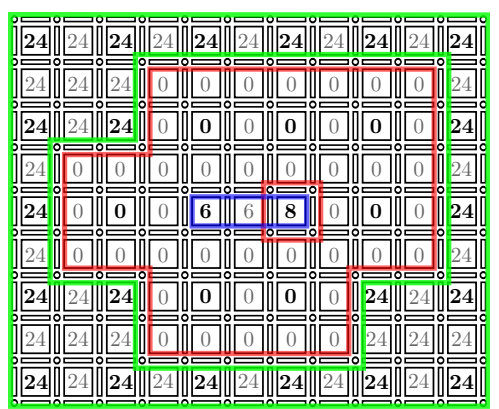

(b) Minimum interpolation.

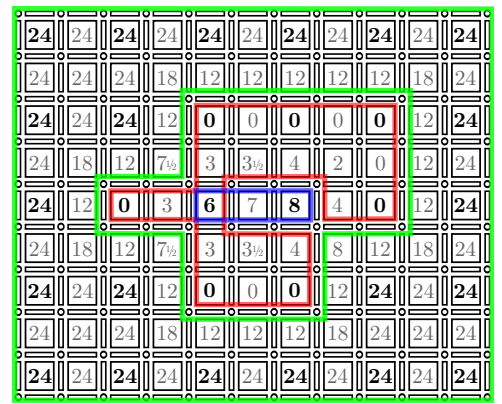

(d) Mean interpolation.

Fig. 4. Original data (a) and (b,c,d) three different interpolations $F$ of the same 2D image that are plain maps. The original image values are depicted in bold in $(\mathrm{b}, \mathrm{c}, \mathrm{d})$; contours of connected components of $[F \triangleleft 7]$ are in red, whereas contours of connected components of $[F \triangleright 5]$ are in green and blue. One can remark that (a) and (d) are not well-composed and that the saturation of the components of (a) and of (d) do not satisfy Lemma 19 and 20, contrary to the components of (b) and (c). In particular, the saturation of the red component in (a) or (d) does not include the blue component.

hereafter. More precisely, we map a function defined on $\mathbb{Z}^{n}$ to a function defined on the Khalimsky grid $H^{n}$ using an adequate space subdivision.

Let us denote by $H_{1}^{n}$ the set of the elements of $H^{n}$ that are open sets:

$$
H_{1}^{n}=\left\{h_{1} \times \ldots \times h_{n} ; \forall i \in[1, n], h_{i} \in H_{1}^{1}\right\} .
$$

With $2 H_{1}^{1}=\{\{2 x, 2 x+1\} ; x \in \mathbb{Z}\}$, we can define several subsets of $H_{1}^{n}$ :

$$
2 H_{1}^{n}=\left\{h_{1} \times \ldots \times h_{n} ; \forall i \in[1, n], h_{i} \in 2 H_{1}^{1}\right\} \text { and } I_{1}^{n}=H_{1}^{n} \backslash 2 H_{1}^{n}
$$

and the subset of $I_{1}^{n}: C_{1}^{n}=\left\{h_{1} \times \ldots \times h_{n} ; \forall i \in[1, n], h_{i} \in H_{1}^{1} \backslash 2 H_{1}^{1}\right\}$.

Let us denote by $\mathcal{M}(\mathbb{Z})$ the set of multisets having $\mathbb{Z}$ as the underlying set of elements; for instance we have $\{1,1,2\} \in \mathcal{M}(\mathbb{Z})$. Let op : $\mathcal{M}(\mathbb{Z}) \rightarrow \frac{1}{2} \mathbb{Z}$ be 
an operator over a multiset of integers that is increasing with respect to any element of the multiset, that is, $\forall z=\left\{z_{1}, \ldots, z_{k}\right\} \in \mathcal{M}(\mathbb{Z}), \forall i \in[1, k]$, we have:

$$
z_{i}^{\prime} \leq z_{i} \Rightarrow \operatorname{op}\left(\left\{z_{1}, \ldots, z_{i-1}, z_{i}^{\prime}, z_{i+1}, \ldots, z_{k}\right\}\right) \leq \mathrm{op}(z) .
$$

Some well-known eligible operators are the minimum, the maximum, and the median $($ with $\operatorname{med}(\{a, b\})=(a+b) / 2)$. From any operator op we can derive an "interpolation" of a function $f: \mathbb{Z}^{n} \rightarrow \mathbb{Z}$ into a plain map $F: H^{n} \rightsquigarrow H^{1}$ :

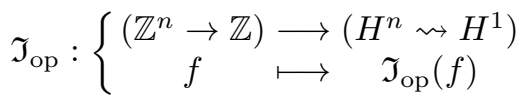

where $\mathfrak{I}_{\mathrm{op}}(f)$ is recursively defined by:

$$
\forall h \in H^{n}, \mathfrak{I}_{\mathrm{op}}(f)(h)= \begin{cases}2 f\left(\frac{h_{1}}{2}, \ldots, \frac{h_{n}}{2}\right) & \text { if } h \in 2 H_{1}^{n} \\ \operatorname{op}\left(\left\{\mathfrak{I}(f)\left(h^{\prime}\right) ; h^{\prime} \in s t_{X}\left(c l_{X}(h)\right) \cap 2 H_{1}^{n}\right\}\right) & \text { if } h \in I_{1}^{n} \\ \operatorname{span}\left(\left\{\mathfrak{I}(f)\left(h^{\prime}\right) ; h^{\prime} \in s t_{X}(h) \cap H_{1}^{n}\right\}\right) & \text { otherwise. }\end{cases}
$$

Furthermore, the elements of $C_{1}^{n}$ shall not lead to extrema in $\mathfrak{I}_{\mathrm{op}}(f)$.

Proposition 24 Any plain map obtained thanks to the max- or the min-interpolation is well-composed.

The max- and min- interpolation reproduces the Rosenfeld 4- and 8- topology. The tree of shapes obtained with this interpolation is the one computed by all previous existing algorithms [7]. However, the max- or the min-interpolation do not lead to a self-dual filtering. This is illustrated in Fig 4.b.

Proposition 25 The median interpolation of a function defined on $\mathbb{Z}^{2}$ leads to a self-dual plain map.

This proposition is illustrated on Fig. 4.c.

Conjecture 26 With the suitable space subdivision, the median interpolation leads to a self-dual map whatever the dimension of the space.

Remark 27 Fig. 4.d shows that the mean operator is not suited for our type of interpolation.

\section{Conclusion}

In this paper, we have shown how to obtain a well-composed plain map from any set of data defined on a grid, and we provide a median-interpolation operator inducing a true self-dual tree. In another paper [10], we provide a quasi-linear algorithm for computing the tree of shapes from a $n$-dimensional well-composed plain map. A dedicated algorithm (less memory consuming) will also be provided for the $2 \mathrm{D}$ case.

Acknowledgements. This work received funding from the Agence Nationale de la Recherche, contract ANR-2010-BLAN-0205-03 and through "Programme d'Investissements d'Avenir" (LabEx BEZOUT n ANR-10-LABX-58). 


\section{References}

1. Alexandroff, P.: Diskrete Räume. Math. Sbornik 2(3), 501-518 (1937)

2. Alexandroff, P.: Combinatorial topology. Dover Publications (1947)

3. Arenas, F.: Alexandroff spaces. Acta Math. Univ. Comen. LXVIII(1), 17-25 (1999)

4. Aubin, J., Frankowska, H.: Set-valued analysis. Birkhauser (2008)

5. Bertrand, G., Couprie, M.: A model for digital topology. Discrete Geometry for Computer Imagery pp. 229-241 (1999)

6. Boxer, L.: Digitally continuous functions. PRL 15(8), 833 - 839 (1994)

7. Caselles, V., Monasse, P.: Geometric description of topographic maps, Lecture Notes in Mathematics, vol. 1984. Springer (2010)

8. Escribano, C., Giraldo, A., Sastre, M.A.: Digitally continuous multivalued functions, morphological operations and thinning algorithms. JMIV 42(1), 76-91 (2012)

9. Evako, A.: Dimension on discrete spaces. International Journal of Theoretical Physics 33, 1553-1568 (1996)

10. Géraud, T., Carlinet, E., Crozet, S., Najman, L.: A quasi-linear algorithm to compute the tree of shapes of $n$ d images. In: ISMM (2013), this volume

11. Gonzalez-Diaz, R., Jimenez, M.J., Medrano, B.: Well-composed cell complexes. In: DGCI. LNCS, vol. 6607, pp. 153-162. Springer (2011)

12. Kovalevsky, W.: A new concept for digital geometry. Shape in Pictures, NATO ASI Series F 126, 37-51 (1994)

13. Latecki, L., Eckhardt, U., Rosenfeld, A.: Well-composed sets. Computer Vision and Image Understanding 61(1), 70-83 (1995)

14. Latecki, L.: 3d well-composed pictures. CVGIP: Graphical Model and Image Processing 59(3), 164-172 (1997)

15. Marchadier, J., Arquès, D., Michelin, S.: Thinning grayscale well-composed images. PRL 25(5), 581-590 (April 2004)

16. Monasse, P., Guichard, F.: Fast computation of a contrast-invariant image representation. TIP 9(5), 860-872 (2000)

17. Nakamura, A.: Magnification in digital topology. In: Klette, R., Zunić, J. (eds.) IWCIA. LNCS, vol. 3322, pp. 260-275. Springer (2004)

18. Ronse, C.: Regular open or closed sets. Tech. Rep. Working Document WD59, Philips Research Lab., Brussels (1990)

19. Rosenfeld, A.: "continuous" functions on digital pictures. PRL 4(3), 177-184 (1986)

20. Siqueira, M., Latecki, L.J., Tustison, N., Gallier, J., Gee, J.: Topological repairing of 3D digital images. JMIV 30(3), 249-274 (2008)

21. Stelldinger, P., Latecki, L., Siqueira, M.: Topological equivalence between a 3d object and the reconstruction of its digital image. PAMI 29(1), 126-140 (2007)

22. Tsaur, R., Smyth, M.: "continuous" multifunctions in discrete spaces with applications to fixed point theory. In: Digital and Image Geometry, LNCS, vol. 2243, pp. 151-162. Springer (2001)

23. Wang, Y., Bhattacharya, P.: Digital connectivity and extended well-composed sets for gray images. CVIU 68(3), 330-345 (December 1997) 